\title{
NURSES' VIEWS OF THE COPING OF PATIENTS*
}

\author{
David L. Kahn, ${ }^{1}$ Richard H. Steeves ${ }^{2}$ and Jeanne Q. Benoliel ${ }^{3}$ \\ 'University of Michigan School of Nursing, 400 North Ingall's, Ann Arbor, MI 48104, U.S.A., \\ ${ }^{2}$ University of Virginia School of Nursing and ${ }^{3}$ University of Washington School of Nursing
}

\begin{abstract}
The findings of a study that explored the beliefs, assumptions and ideas nurses have about the coping of patients are presented. Interactive interviews with 26 nurses were used to elicit explanations of the meaning of coping and stories from their practice that illustrated coping. Analysis of the interview transcripts revealed three themes in the form of idioms or particular and different ways of talking about coping. Each idiom represented a different perspective or view of coping. The first idiom represented a view of coping as a rational, cognitive problem-solving response to illness. The nurses attributed, and thus valued, this view to science. In the second idiom the nurses spokc of coping as permeated with values that contrasted with the prior view of coping as a rational process. In the final idiom the nurses spoke of coping as courage-they told stories of patients who had faced existential situations with strength and will. The focus of this idiom was on issues of spirituality, struggle, personal meaning and acceptance.

After each idiom is delineated and illustrated by data, the discussion is concentrated on the orientational and ontological metaphors that underlie them. Interpretation of the origin and construction of these different ways of talking about coping, and their underlying metaphorical meanings, is made in the context of cultural and subcultural influences.
\end{abstract}

Key words -coping, metaphors, nursing practice, meaning, qualitative research

Coping is an important subject in most of the clinical arenas in which nurses practice. Every day in such settings nurses attend to, assess and evaluate the coping of their patients, talk about this coping among themselves and with other practitioners, and comment upon and document evidence of coping in clinical records. In our experience, students of nursing usually begin speaking about the coping of assigned patients during their first clinical rotation, incorporating the notion into their earliest care plans. On the other hand, what coping means in a broader and deeper sense - in terms of why it is attended to, expected and apparently valued by nurses - remains tacit, part of a larger system of cultural beliefs that nurses understand, share and take for granted.

These observations are interesting in that extensive use of the term, coping, historically is a relatively recent development in the language of nursing practice. For example, coping was first indexed in the Cumulative Index to Nursing and Allied Health Literature in 1979, encompassing material formerly indexed under the terms, defense mechanisms and, especially, psychological adaptation. Prior to 1979, coping appeared infrequently in journal titles. In 1979 25 articles were indexed under the heading of coping; 10 years later that number had increased more than fourfold to 107 entries.

Presumably, the increasing popularity of coping in the nursing literature has been related directly to the

*An earlier version of this paper was presented at the Veronick Conference, University of Virginia School of Nursing, Charlottesville, Virginia, in April, 1989. influence of the stress and coping paradigm on all the health sciences over the past 25 years. This paradigm, set forth in the classic text by Lazarus, defines coping as a response to stressors in terms of both problem solving and emotion regulating behaviors [1]. Nursing research has tended to remain true to the theoretical assumptions of this paradigm. For example, the widely used Jalowiec Coping Scale identifies and measures three dimensions of coping derived from the stress and coping paradigm [2]. It is questionable, however, whether in nursing practice the everyday use of the term, coping, is grounded as firmly in specific theory. Thus, the purpose of this study was to describe the beliefs, assumptions and ideas nurses held about the coping of patients.

\section{METIIOD}

The data described in this paper were collected as part of a larger study on the language of nursing practice. Besides coping, the investigators also examined the beliefs of nurses about the meaning of caring and suffering as well. Those findings were reported in previous papers $[3,4]$. This section briefly reiterates the interpretive method of the larger study. Subsequent discussion of findings, however, is focused on data that have not been published previously.

The interpretive design of the study was influenced by two scholarly traditions. One influence was cognitive anthropology, especially as illustrated in the work of Spradley and Agar [5-8]. This perspective provided a guiding theoretical assumption that beliefs and ideas about coping that nurses shared as a 
cultural group would be embedded in their discourse on the subject, in other words, in the way they used language in thinking and talking about coping. The second influence was phenomenological as illustrated particularly in the work of Benner $[9,10]$. This influence led to an emphasis on the collection of clinical narratives about coping as a way to access the knowledge of nurses about this aspect of clinical practice.

\section{Sample}

A purposive sample of 26 incoming graduate students of nursing were interviewed. Participants were self-selected, recruited by a letter of invitation and required to have practiced nursing at least one fulltime year. Graduate nursing students were sampled for two reasons. First, such nurses were not thought to be significantly different in their subcultural perspective from other nurses, since they were just beginning graduate study. It was expected, though, that they would be particularly articulate about their experiences in nursing practice. Second, as Schulz theorized, members of a group in transition between two different social and cultural identities are apt to be especially reflective on the identity recently relinquished [11]. The limitations of such selection for generalization to all nurses are obvious and go beyond the nonrandom nature of the sample as most nurses in the United States do not enter graduate school. Procedures to protect the rights of the participants and maintain confidentiality were integrated throughout the study and approved by a university committee for the protection of human subjects.

The participants' mean age was 32.1 years $(\mathrm{SD}=4.8)$. The mean of practice experience was 7.6 years $(S D=4.1)$, ranging from 2 to 16 . The clinical background of the nurse was diverse. Practice experiences were reported in a wide variety of settings, including intensive care, pediatric, medical, obstetrical, surgical, psychiatric, home health, hospice, public health and long-term care. Nineteen participants reported practice experience in at least two different settings. Six of the 26 participants were males.

\section{Data collection}

Data were collected by means of an audiotaped interview conducted by either of the two co-primary investigators (the first and second authors). Refinement of an interview guide and procedures to ensure across interviewer reliability and content validity have been described previously [3, pp. 203-204]. Interviews were structured to elicit data in three content areas:

(a) a list of words and phrases used synonymously with coping and contextual description of such use;

(b) the semantic principles underlying the meaning of coping; and

(c) prototypic examples from clinical practice that illustrated coping.
The first two content areas were found to be useful for understanding suffering and caring, but only the third content area, clinical examples, were useful in coming to an understanding of coping and are the source of the data that are presented here.

In order to elicit these clinical examples, the nurse informants were asked to talk about a patient who came to mind when they thought about coping. All of the informants were able to recall a patient and tell the story of that patient. The nurses demonstrated considerable variability in their approach to relating these narratives. Some stories were precise and colorful in their detail and others were sketchy and consisted primarily of explanatory comments about the patient's behavior. All these narratives and the attached explanations were recognized as creations of the nurse informants and indicative of their beliefs and understanding and not of the patients about whom the stories were told. The interviews were interactive in the sense described by Morse in that participants were not discouraged from adding tangential material, and interviewers shared their ongoing interpretations of the participants' remarks for validation and clarification [12].

\section{Data analysis}

After all taped interviews were transcribed and checked for accuracy, hermeneutical analysis was begun following procedures described by Kockelmans [13]. The analysis took place in three phases. First, statements from each nurse informant's narrative that demonstrated some aspect of coping were extracted in the most parsimonious form that retained contextual meaning. These statements were descriptions of or valuative comments about the actions or qualities of characters in the narratives. These statements remained in the exact words of the nurse informants. For example, a statement that was extracted from one informant's story was "She was coping by being psychotic. Maybe by freaking out, that was how she was coping with this awful event (the death of a family member)."

In the second phase of analysis, these verbatim statements were grouped according to observed similarities. These groups were researcher devised since the informants were asked to relate narratives and not analyze their experience. However, the grouping of statements was based on the researchers' interpretation of the nurse informants' ways of understanding their experiences and not on a theoretical framework brought in from the outside. Each group was labeled as a category and the rules for inclusion in that category were defined. To continue with the example above, the statement was assigned to a category termed 'extreme behavior as coping'.

Finally, the categories were grouped into themes. At this level of analysis the researchers moved beyond an attempt to understand how individual participants understood their experiences and how these understandings might be similar or contrasting and moved 
to making general statements concerning how the whole group of nurse informants might be characterized concerning its beliefs about coping. Thus, the themes and relationships among themes served as the basis for interpretation of the meaning of the data as a whole. The example above and the other statements in that category helped constitute the theme 'coping as a value', which is discussed below.

Throughout each phase of analysis, intact interviews were reexamined and compared with categorical data to validate interpretations. Several checks to reduce investigator bias and ensure validity of study findings were used. Each investigator separately coded the verbatim statements from about $1 / 3$ of the transcripts, randomly assigned. No discrepancies were found in the amount or kind of statements coded by each investigator when compared. Second, the grouping of verbatim statements into categories was done together and required agreement between both investigators, thus providing some consensus validity. All disagreements were resolved through appeal to the original transcript and the context of the statement therein. Finally, the remaining $1 / 3$ of the transcripts were coded, and categories from this data were compared with the previous categories. No discrepancies were noted at this point. All categories were noted in a minimum of six transcripts. All categories existed in interviews conducted by both investigators.

Two colleagues were asked to match randomly selected verbatim statements with the categories and inclusion rules. Both judges exceeded a predetermined criterion level of $90 \%$ agreement with the investigator's schema.

\section{FINDINGS}

It must be kept in mind that the data constituted nurses' narratives about the coping of their patients. None of the data were derived from patients, and therefore the analysis was aimed at reaching an understanding of the nurses' bclicfs about the coping of their patients. The findings are revealing of nurses and not of patients.

Analysis of the data produced three different themes around which categories derived from the informants' discourse on coping clustered. The discussion in this section, for the sake of brevity, is focused on the themes instead of the individual categories. Each theme can be viewed as a different idiom or way of speaking about coping that glossed a different set of ideas and beliefs nurse informants held on the subject. The themes were not mutually exclusive; most informants expressed beliefs that were categorized in more than one theme. Thus, the structure of the three themes does not represent a static view of coping held by this group of nurses, rather it represents a map of the terrain over which their ideas and beliefs about coping ranged in their discussions of the subject.
In the first theme the nurse informants spoke of the coping of their patients in terms of the expected scientific paradigm: coping was a problem-solving process their patients went through. In the second theme, nurse informants spoke about value-laden and moral aspects of coping. In the final theme, coping was spoken of on a different level and in a different way entirely. In this theme the discourse on coping became existential, and informants spoke primarily about certain patients who had coped in unique, meaningful and courageous ways.

\section{Rational problem solving}

In the first theme nurse informants understood and spoke about the coping of their patients in a way that might be termed a 'received view'. This view was received in that it was attributed to the external authority of science. The informants were aware that a particular perspective of coping dominated nursing literature on the subject and were reflexive about this view as valued in the scientific environment they had just entered as graduate students. In a typical statement a nurse informant described coping as a series of cognitive steps and then said, "of course the reason I came back to school was to understand better how these [kinds of] things work". In this perspective, coping was understood as a problem-solving process with cognitive and behavioral components and with a functional goal. Comments such as "[coping is a method to continue to function with ... a problem", "people that cope, plan and do things that [let] them work through their problem", and "coping implies a cognitive process with behavioral outcomes [and] finding ways to function" were typical. An underlying assumption held by informants was that coping stemmed from a 'conscious' decision to cope and required 'cffort' and 'work' on the part of the patient. Indeed, the notion of effort, or 'trying hard' as one informant put it, was the dominant metaphor in this theme.

The nurse informants believed that as a conscious process coping involved a scries of distinct stcps or stages. Although the exact labels varied, most informants identified three stages and were in general agreement about the order in which these stages occurred. In order to cope with a problem a patient first had to accept that the problem existed. As one informant phrased it, "coping, I think it goes through stages, [and] first you have to accept what's going on". Similarly, from this perspective not coping meant that a patient 'hasn't accepted what's going on'.

According to informants, the second step in this process involved making plans and seeking information. For example, one informant said, "a person must reach some level of realization that something isn't working [in order to cope, and] then it means planning and working through the situation". In discussing this step informants most often spoke of the nursing role. Statements like "I do a lot of 
teaching with my patients because I find that the more information they have, the better they cope" were typical.

The final step in the process of coping was for the patient to choose an appropriate response to the problem from a repertoire of strategies and resources. As one informant noted, it was important for patients "to learn lots of different coping behaviors so that [they] could choose the best one for their situation". These strategies were spoken about both specifically and generally as the following examples illustrate.

[Speaking of a patient who lost a leg] I would try to get [the patient] to accept facts, that they're limited in their ability to do certain things ... they have to learn, get the information they need so that it doesn't limit them from functioning ... [then] get a prosthesis.

They say 'Ok, you know I've got CA, ok I've got CPD'. They've accepted it. Then they're on the way to beginning to cope because they're gonna get information on ... their limitations and how to adjust to these limitations ... what to do to continue to function.

\section{Coping as a value}

The nurse informants' understanding of the coping of their patients as a problem-solving process was the explanation of coping informants invariably advanced first. As informants continued to discuss coping, most of them identified another view and began speaking of coping in another way. In this idiom coping was described as a value of nurses or as a value of patients.

For the nurse informants, coping was a value of nurses (and presumably the larger health care system) to the extent it was equated with patient compliance and cooperation. The coping of patients was evaluated in terms of whether or not their behavior conformed to the nurses' expectations. For example, an informant noted that whether or not a patient was coping was 'a value judgement'. Another nurse informant offered a story about an adolescent girl who had coped 'badly' during labor and delivery of her baby and explained this judgement with the statement: "She was not doing what I as the nurse wanted her to do". In another example about a COPD patient a nurse informant stated:

I thought he wasn't coping as well all the time that we were trying to wean him [from a ventilator]. ... But that's probably because he wasn't doing what we [the nursing staff] wanted him to do.

Another nurse said that she evaluated coping in terms of "whether [patients] are getting to the doctor or not" and following medical orders. Other informants spoke of this aspect of coping in terms of "how actively' the patient 'participated' in the plan of care with active participation opposed to 'denial' and 'withdrawal'. Another group of nurse informants evaluated coping in terms of the control patients were able to maintain over their own behavior. For these nurses, patients who coped were, in a typical statement, people "who remained in control no matter what's happening to them ... [because] at a certain point some people are better able to cope with things".

Most informants regarded this view of coping as unattractive or socially undesirable but realistic. As an informant explained:

Coping can mean, well we nurses sometimes use it to mean that they're accepting their treatment or their drugs with little or no questions. Rather than refusing their treatment In real life it's like they're cooperating, they're not resisting, ... we, nurses, have expectations.

In the same vein, other informants noted that 'noncopers' consumed more nursing time, were more 'frustrating' to care for and were 'exhausting'.

Most of the nurses also realized that patients might have a set of values that were different from their own and that patients perceived as not coping well based on the nurse's own values could be viewed as coping under a different set of values. For example, a nurse informant who had first described coping as a typical sequence of problem-solving steps, including acknowledging a need for help, arranging for it and then choosing adaptive behaviors, admitted that:

You can't really tell if someone is coping well. Someone could be happy and doing well but deep down going through a lot of anguish ... Also, some things like anger could be not coping, maladaptive. [But] sometimes anger can be coping.

Another nurse informant who had stated that "it was important [for patients] to learn lots of different coping behaviors so that [they] could choose", next pointed out that behaviors she usually identified as 'noncoping', including 'withdrawal' and alcohol use, could also be viewed as ways some people coped. The problem from this perspective was that "coping was fairly subjective ... [and] depends on the [patient's] own definition".

Nurse informants were aware of the ironies involved in this view of coping. For example, with laughter an informant said of conflict between a patient and herself, "her definition of coping was different than my definition of coping, and I wasn't coping because of it". Another nurse noted that some patients seemed to cope by not coping and offered the story of a woman with leg ulcers who, she believed, coped with her problem by 'letting the ulcers go' so they would deteriorate and she would be admitted to the hospital. Similarly, another informant, in the statement which was used as an example in the above discussion of analysis methods, insisted that one of her patients had coped by displaying behavior that would usually be classified as the ultimate example of failure to cope: "She was coping by being psychotic. Maybe by freaking out, that was how she was coping with this awful event".

\section{Coping as courage}

In the final theme the discourse took on a different form and tone altogether. Nurse informants did less explaining while telling their stories and relied instead 
on vivid descriptions of patients. The meanings these vivid narratives conveyed can be interpreted as describing an alternative view of coping.

The nurse informants spoke about what might also be termed courage-coping as the strength or will their patients displayed in the face of their own suffering and dying to persevere, confront, endure and accept difficulties. Informants described issues of spirituality, personal meaning, struggle and acceptance as the basis of this different perspective of coping.

Coping in a spiritual way had nothing to do with problem solving from this perspective. For example, an informant told the story of "Walt, the principal of a local high school who had leukemia". As she explained, Walt could do nothing to solve this problem other than wait "a long time for his white blood count to come up". In this case planning had no role in coping. Instead, Walt "faced his illness ... because he was pretty religious". Another informant noted in a similar story that her patient coped through religion while rejecting 'biomedical' information about her situation, for this person it was 'important' simply to 'say that it's God's will'.

Coping in a spiritual way also included a different perspective on moral value and virtue, one in which nurses' expectations of behavior were moot. For example, an informant offered the story of a man, 'the father of three', who was paralyzed in a motorcycle accident. He remained on the nursing unit for a 'long time' and was a 'very difficult patient' because 'he had so much anger'. Yet, the informant said:

He coped through religion. He hadn't been religious [before the accident] but a family member brought in a mustard seed. He had to grab onto that ... so I think he was coping.

Similarly, another informant described a patient and her coping in this way:

She was a truck stop woman. It's like a waitress who lives a tough kind of life. Hangs around, you know, drinking beer. She was happy with that. And yet she had this real holy Christian side of her. When she was sick ... she would play her tapes of religious music and all that sort of stuff... just to give her relief.

Other informants told stories of patients in which coping had to do with their "making sense of the[ir] fortune, making sense of what it is in some way, making sense of whatever you are confronted with". In these examples, coping was equated with seeking meaning in confronting the existential situation. This created a very different notion of effort than advanced by informants when coping was viewed as a problem-solving process. For example, as an informant generalized after telling the story of a specific patient who died of cancer:

I have seen people who have tried and done everything they could and they still die. But at the end when you talk to them and they know that they are going to die, they will tell you [like this patient did] they felt they have done everything they could do. They say, 'I've tried' [and] that [the] real big thing for them to know [is] that they have tried.
Indeed, many of the informants' stories associated with this view of coping involved patients who confronted their illnesses and endured courageously. Although the word, courage, was not specifically used, it is evident in the narratives of these nurses about their patients' coping. For example, an informant talked about the coping of an ill husband and his wife:

A guy we admitted this weekend-he and his wife just totally amazed me. He's got cancer of the throat and mouth and he's about 64 I think. And it's been going on for about three years. The tumor is just eroded itself so his whole chin and his mouth is just open ulcerated tumor. So the feeding tube was plugged up, so his wife, she wasn't able to get any pain meds or fluids into him. She and he have been living with this at home for this whole time. ... She suctions him and they both deal with this at home. He didn't want to come into the hospital. The only way we were able to convince him to come into the hospital was so that she could get some rest. And I mean that's coping. Like nothing short of death was gonna stop them from going on.

A similar example from another informant about a woman with COPD was just as vivid:

She was blue all the time. I mean when you listened to her lungs you couldn't hear air moving. But she got around somehow. She had an electric wheelchair that she would get in and go down the street on; she'd take her inhalers. She was tring to stand up and reach something on the [grocery store] shelf. She's just as frail as can be from all her steroids that she's on. [She was] standing on her wheelchair trying to reach that can of whatever she wanted. Fell off. Broke a rib. Crashed right in the middle of Safeway. And yet it was important for her to not ask for help. She coped [like that] a really long time beyond all odds. If I'd seen her in the hospital clinically, l'd say this woman could never make it at home.

A final aspect of the nurse informants' alternative view of coping was a revised notion of what acceptance meant. In this idiom, like the notion of effort, acceptance was redefined in existential terms. In some stories told by informants, instead of accepting that they had a problem to solve, some patients coped by accepting that their situation had no solution and courageously proceeding with their lives from that point to whatever extent possible. For example, an informant said of a patient with liver cancer:

All of sudden he realized he wasn't going to get better. Just like that he started getting ready to die, really enjoying time with his wife. He was coping.

Another informant described a patient with severe, chronic pain:

There was this guy with a lot of pain. He couldn't get out of the hospital. He accepted his pain and that was why he was so helpful to some of the other patients. He was almost like an employee. There was some part of him that had nothing to do with his pain.

Finally, this perspective on coping is perhaps exemplified most completely in the following story. This nurse informant told of a religious man who confronted his illness, courageously struggled against it and ultimately accepted it and died:

In the VA [hospital] we had a guy that was on the ventilator. 
He'd had CA and he had surgery, but then he had a trach [put] in. He was doing real good, and he was real positive about getting well. Then he went bad. He'd always been real positive [because of his faith in God] about doing everything to get well. But he said no about the ventilator, but his [blood] gases were bad so they put him on anyway. Then we could never get him off. He tried-we had him in the MICU for months. We'd take him off, and he'd last maybe two days, and he'd go back on. He went on and off for several months. Most of this time he wanted to be on it. We had to fight with him to even try to wean him off. But finally he was the one, with perfectly good [blood] gases, who said turn it off. He lasted about a week. That's an extreme way of coping.

\section{DISCUSSION}

The findings show that the language these nurses used to tcll storics about patients' coping reflected three different cultural sources of ideas gained through nursing school, experience as a practicing nurse and the values of Western culture as a whole. Thus, the following discussion is not so much about the content of the nurses' narratives as it is about the cultural sources of meaning the nurses drew upon to make sense of them would. The source for the first theme of coping as rational problem solving fits with the scientific training nurses received, which is exemplificd in the increased use of the Lazarus coping paradigm in nursing curricula in the 1980s. The second theme of coping as value-laden (and not always rational) wherein patients' behaviors were described as contradictory to their interests or demonstrated an irrational approach has its source in nursing practice and the social context of working nurses. The third theme of coping as courage has its sources in deeply held beliefs current in Western culture.

In a sense these first two meanings ascribed by nurses to patients' coping behaviors comprise a dialectic between the 'ideal' of coping exemplified in rational problem solving and the 'reality' of coping shown in personalized, sometimes emotional modes of response observed in actual experience with patients. For both forms of coping the nurses had formulated explanations to account for the observed behaviors, the differences between them and some reasons that both forms of coping might be used by the same patient. The nature of the dialectic between these two meanings is fluid.

Much in contrast, the nurses' discourse on coping as a demonstration of courage centered on the ways by which patients responded to difficult illness situations and gave meaning to these situations. In the examples illustrating this theme, the nurse's attention was not focused on the patient's problem-solving behaviors. Rather, coping was perceived by the nurses who described these incidents as an admirable way of enduring and responding to tragic life circumstances. In this formulation of coping, the focus is on the patient as a person. The data suggest that this perspective on coping (unlike the other two) is depen- dent on contexts in which patients are facing major existential concerns. Further research is needed to evaluate the validity of this claim.

The finding that coping had multiple meanings within the discourse of these nurses suggests a complex relationship between the world of ideas in nursing and the world of patient care situations. The three themes or idioms identified in the data might be considered reflections of different metaphors through which coping as a human phenomenon is described and explained. According to Lakoff and Johnson, the conceptual systems governing human thoughts and actions in everyday life are fundamentally metaphorical in nature and operate most of the time without conscious awareness [14]. These orientational and ontological metaphors are learned out of human experiences in the physical and social world and reflect the values of particular cultures and subcultures. We speculate on how cultural and subcultural experience has contributed to the origins of these different ways of talking about coping.

\section{Cultural and subcultural influences}

Exposure to the stress and coping paradigm of Lazarus through nursing instructional programs was identified earlier as one influence on nurses' ideas [1]. Coping as problcm solving in response to illness situations was strengthened in nursing discourse in the 1980 s by research on patient coping and studies on nurses' responses to stressful work [15-17].

Although the nurses attributed their views to the influence of science, the idea of coping as a way to solve problems likely had origins in their earlier everyday experiences as Americans with what Lakoff and Johnson have called the 'problems are puzzles' metaphor [14, p. 145]. In this conceptual orientation, problems can be solved when individual thought and effort are directed toward finding the pieces of the puzzle and putting them together correctly. Not surprisingly, this perspective on problems carries within it the Cartesian view of mind-body separation, the primacy of rationality over sensation, the centrality of facts to goal achievement and the importance of individual effort.

In the culture of the United States, high value is attached to individualism and personal achievement of goals through hard work and persistence. The importance attached to these concepts can be traced historically in the belief systems of the four major American traditions - biblical, republican, utilitarian individualist and expessive individualist - that contributed to the current middle-class culture [18].

One societal manifestation of the value accorded to individual effort is the large number of 'how to do' books available to lay readers in newsstands and bookstores across the country. These prescriptive treatises (many in paperback editions) offer guidance and direction for all manner of life's problems-from building book cases and fixing cars to losing weight, recovering from divorce and raising children. It is 
probable that the nurses in this study were initiated into thinking about problem solving as process long before they entered the nursing subculture. Indeed, in some respects the coping process identified in their language reflects what Benner and Wrubel have called the popularized view of coping as self-management strategies that emphasize control and distancing behaviors as solutions to stress-related problems [19].

From these data we surmise that the everyday work of nurses is guided by the high value accorded to the 'problems as puzzles' metaphor as a model of patient coping $[14$, p. 145]. Indeed, the nurses spoke of their activities with patients primarily as cognitive interventions to assist patients in gaining information to solve the illness-related puzzles they faced.

Yet to judge from our findings, their contacts with patients put them into situations that triggered thoughts about coping as other than rational mental activity. As Lakoff and Johnson [14, p. 19] have argued, concepts can be understood in terms of more than one metaphor, and different experiences stimulate different ways of thinking about the concept of interest. In Ricoeur's view, new meanings are created in response to events in which earlier meanings are contradicted or brought into question [20].

One way to reconcile contradictory meanings is to use a naming process whereby those who fit the definition and those who do not are given opposing labels - in this case, problem solvers are copers whereas those who behave otherwise are noncopers. Another way to reconcile differences is to ascribe meanings that carry valuc judgements, implying that one behavior is better than another.

The nurses' views on coping as representing opposing images suggest that the meanings were embedded in the UP-DOWN orientational metaphor which Lakoff and Johnson believe is a central conceptual system guiding everyday human activities. In this perspective, the spatial orientation of UP is associated with being conscious, being healthy and alive, having control, being rational and being virtuous or good. In contrast, DOWN is associated with being unconscious, being sick or dead, lacking control, being emotional and being depraved or bad [14, pp. 14-19].

The nurses' terminology for coping as a value includes images representing the DOWN orientation of irrational behavior and nonvirtuous action. Such behaviors were classified as 'bad' in contrast to those behaviors in which patients did what the nurses expected and wanted. The assignment of 'good' and 'bad' to opposing views on the meaning of coping supports the position that values are embedded in cultural orientational metaphors, and these values (generally implicit) guide the priorities and standards of the people living there. To judge from these findings, within the work culture of nursing, patients' compliance with nurses' recommendations is equated with good coping.

Through work experience the nurses became aware that patients responded to illness situations in a variety of ways that made the work of nurses difficult and frustrating. Thus, 'bad' coping was attributed to patient behaviors that interfered with the nurse's abiliy to cope with patient care problems in a way satisfying to the nurse.

What is striking about the views of coping as problem solving and coping as a value is that the nurses' words consisted usually of behavioral descriptions of patients' actions as they were cognitive or emotional precesses that operated regardless of the patient's situation or context. In this regard, they might be considered as manifestations of the 'mind as machine' metaphor in which cognitive structures determine a person's understanding of the world and choice of behaviors [20, pp. 29-40]. Rather in contrast, the nurses' views of coping as courage incorporated the patient's situation into an image that conveys a phenomenological perspective on coping as "what people do when personal meanings are disrupted" $[20$, p. 408].

In the narratives that were used to display the theme of coping as courage, the nurses described patient situations that conveyed a picture of people living with courage and endurance in the face of adversity. Although the specifics faced by different patients varied, these narratives have some common themes. They showed patients in tragic life circumstances, facing tough decisions without neat outcomes, and drawing on inner resources to find strength and to create new meanings out of adverse circumstances.

These stories do not provide a sense of coping as mental effort to solve problems. Rather, they convey images of individuals responding as integrated beings to major impingements that altered the meaning of their daily lives and brought many facc-to-facc with the existential questions of life and death. Also clear in these narratives, the nurses admired these patients for the ways that they chose for responding to their adversities and the changes imposed by them. These stories of paticnts who confronted illness and death with courage are representative of the powerful heroic myth of individualism--exemplified in the United States by the pioneer who struggles alone against all odds [18, p. 144]. In other words, the essences of these stories can also bc capturcd in a cultural metaphor, COPING IS HEROISM.

\section{Interview as discourse}

It is difficult to evaluate from these data how much the nurses had reflected on the meaning of coping prior to the interviews. It is conceivable that they had incorporated interpretations of coping out of their lived experiences as nurses in a work world in which these terms are part of the taken-for-granted frame of reference of the subculture. If such is the case, they form part of what Schutz has called 'prepredicative thinking'-knowledge taken on without analytic thought about its meaning [21, p. 120]. 
On the other hand, it is plausible to speculate that the interview functioned as discourse through which the nurses disclosed to themselves other meanings of coping. In this case, the interview provided opportunity for the personal story to serve as a method of discovery of new meaning, similar to the outcomes described for women's consciousness-raising groups [22, p. 115]. In Ricoeur's way of thinking, the dialectical exchange of ideas can lead to a shift in contextual thinking such that the meaning of a word is revised by means of a new metaphorical lens [21]. Conceivably, the interview served as discourse through which some of the nurses came to interpret coping in a new frame of reference.

\section{CONCLUSION}

Although these findings cannot be generalized to the language meanings of nurses in general and certainly not the meanings of patients since the narratives were the nurses' constructs of the patients' experiences, the findings nonetheless provide evidence that the language of nursing is embedded in the language of the larger culture. With reference to patient behaviors, coping carries different meanings, depending on the metaphorical frame of reference through which it is viewed. It is probably fair to say that the meanings of coping derived from this analysis do not reflect the influence of science so much as they do the values and beliefs of middle-class American society as these have become incorporated into the conceptual systems through which nurses experience the work of nursing and give meaning to behaviors of the patients they encounter.

Acknowledgements - The authors acknowledge the financial support of Sigma Theta Tau, Psi Chapter, Seattle, Washington, for this study.

\section{REFERENCES}

1. Lazarus R. S. Psychological Stress and the Coping Process. McGraw Hill, New York, 1966.

2. Jalowiec A., Murphy S. and Powers S. Psychometric assessment of the Jalowiec coping scale. Nursing Res. 33, 157-161, 1984.

3. Kahn D. L. and Steeves R. H. Caring and practice: construction of the nurse's world. Scholarly Inquiry for Nursing Practice 2, 201-216, 1988.

4. Steeves R. H., Kahn D. L. and Benoliel J. Q. Nurses' interpretation of the suffering of their patients. Western J. Nursing Res. 12, 715-731, 1990.

5. Agar M. H. The Professional Stranger: An Informal Introduction to Ethnography. Academic Press, Orlando, FL, 1980.

6. Agar M. H. Speaking of Ethnography. Sage University Paper series on Qualitative Research Methods (Vol. 2), Beverly Hills, CA, 1986.

7. Spradley J. The Ethnographic Interview. Holt, Rinehart, and Winston, New York, 1979.

8. Spradley J. Participant Observation. Holt, Rinehart, and Winston, New York, 1980.

9. Benner P. From Novice to Expert: Excellence and Power in Clinical Nursing Practice. Addison-Wesley, Menlo Park, CA, 1984

10. Benner P. Quality of life: a phenomenological perspective on explanation, prediction, and understanding in nursing science. Adv. Nursing Sci. 8, 1-14, 1985.

11. Schutz A. Collected Papers: The Problem of Social Reality. Martinus Nijhoff, The Hague, Netherlands, 1962.

12. Morse, J. M. (Ed.) Qualitative Nursing Research. Aspen Press, Rockville, MD, 1989.

13. Kockelmans J. J. Towards an interpretive or hermeneutic social science. Graduate Fac. Philosophy J.: New School Soc. Res. 5, 73-96, 1975.

14. Lakoff G. and Johnson M. Metaphors We Live By. University of Chicago Press, Chicago, 1980.

15. Johnson J. E. Coping with elective surgery. A. Rev. Nursing Res. 2, 107-132, 1984.

16. Jacobson S. F. and McGrath H. M. (Eds) Nursing Under Stress. John Wiley \& Sons, New York, 1983.

17. Lyon B. L. and Werner J. S. Stress. A. Rev. Nursing. Res. 5, 3-22, 1987.

18. Bellah R. N., Madsen R., Sullivan W. M., Swidler A. and Tipton S. M. Habits of the Heart. University of California Press, Berkeley, CA, 1985.

19. Benner P. and Wrubel J. The Primacy of Caring. Addison-Wesley Publishing, Menlo Park, CA, 1989.

20. Ricoeur P. Hermeneutics and the Human Sciences. Cambridge University Press, New York, 1981.

21. Schutz A. On Phenomenology and Social Relations. University of Chicago Press, Chicago, 1980.

22. Becker C. The Invisible Drama. Macmillan, New York, 1987. 